\title{
Influence of Magnetic Moment on Single Atom Catalytic Activation Energy Barriers
}

\author{
Chloe Groome ${ }^{1} \cdot$ Huong Ngo ${ }^{1} \cdot$ Jie $^{2} i^{2} \cdot$ Chen Santillan Wang ${ }^{3} \cdot$ Ruqian $\mathrm{Wu}^{2} \cdot \operatorname{Regina~Ragan~}^{1}$
}

Received: 18 March 2021 / Accepted: 12 July 2021 / Published online: 20 July 2021

(c) The Author(s) 2021

\begin{abstract}
Design of the molecular environment of single atom catalysts (SAC) is promising for achieving high catalytic activity without expensive and scarce platinum-group metals (PGM). We utilize a first principles approach to examine how the spin state of the SAC and reactants can affect catalytic energy barriers of V, Fe, Mo, and Ta on two different graphene defects with differing magnetic moments. Spin polarized projected density of states and climbing image nudged elastic band calculations demonstrate relatively lower activation energy barriers for systems with higher spin state asymmetry near the Fermi energy; $\mathrm{CO}$ oxidation on Ta and V SAC have decreases in activation barrier energies of $27 \%$ and $44 \%$, respectively.
\end{abstract}

\section{Graphic Abstract}



Keywords Single atom catalyst $\cdot$ Spin catalysis $\cdot$ Density functional theory $\cdot$ CO oxidation $\cdot$ Modified Eley-Rideal pathway $\cdot$ Nudged elastic band $\cdot$ Bader charge analysis $\cdot$ Earth-abundant transition metals $\cdot$ Magnetic graphene defects

Regina Ragan

rragan@uci.edu

1 Department of Materials Science and Engineering, University of California, Irvine, Irvine, CA 92697-2575, USA

2 Department of Physics and Astronomy, University of California, Irvine, Irvine, CA 92697-4575, USA

3 Sandia National Laboratories, Livermore, CA 94550, USA

\section{Introduction}

Single atom catalysis is widely studied to minimize the amount of catalyst material while simultaneously maximizing performance. Consider that the expense and scarcity of platinum group metal (PGM) catalysts represents a major economic and manufacturing roadblock to the adoption of fuel cell electric vehicles [1-3]. The PGM loading for a hydrogen fuel cell is typically 10 times higher than the amount present in a catalytic converter. Pt single atom catalysts (SAC) have exhibited increased catalytic mass activity by approximately 37 times when compared to state-of-theart Pt/C catalysts for the hydrogen evolution reaction [4]. As 
only surface atoms significantly contribute to performance, SAC is the most efficient possible use of chemically active material $[5,6]$ and hence of enormous economic relevance to a wide range of industrial applications.

Yet the term single atom catalysis may be misleading, because the molecular coordination of a SAC is of crucial consequence to stability and chemical activity [7-9]. Both the presence of coordinating ligands and the support material have been shown to strongly influence catalytic activation energy barriers [10-13]. Many investigations have focused on the role of the relationship between electronic charge transfer and catalytic energy barriers [12, 14-18]. Recent work has shown that magnetic moment also plays a role in the catalytic reactivity of $\mathrm{O}_{2}$, which is unusually stable with a triplet ground state [19]. Distinguishing the effects of spin state from more traditionally investigated mechanisms such as charge transfer requires understanding the spin polarized density of states of SAC environments, which is most readily accessible with a first principles approach. While advances in electron microscopy imaging and spectroscopy through techniques such as monochromated scanning transmission electron microscopy coupled with electron energy loss spectroscopy (STEM-EELS) [20] and electron magnetic circular dichroism (EMCD) [21, 22] may enable the correlation of not only local electronic but also magnetic structure with atomic structure, the huge range of stabilizing surface defects and transition metal SAC candidates presents a challenge in terms of throughput. Hence, we present a computational materials discovery approach to identify especially promising SAC candidates. Specifically, we investigate SAC composed of transition metals on two structurally similar graphene defect moieties. The single vacancy has a significant localized magnetic moment, while the pyridinic $\mathrm{N}$ defect moiety does not. Our ability to produce high surface area, porous 3-dimensional bicontinuous turbostratic graphene scaffolds, with morphology that facilitates efficient mass transfer as well as simultaneous high electrical conductivity that is important for a catalytic support [23-26] further motivates identification of earth abundant transition metals and fundamental mechanisms in order to inform the design of molecular dopants and adatoms on graphene that optimize catalytic activity.

Chemical reactions undergoing spin inversion have been considered to be spin forbidden or too slow to be practical [27]. Recently, first principles calculations on $\mathrm{O}_{2}$ dissociation facilitated by gold and silver clusters demonstrated that spincrossing is more likely when spin states are close in energy. Specifically, clusters with odd and hence, unpaired electrons, exhibited energy barriers that were lower than systems with even number of electrons and this was attributed to spincrossed pathways [28]. The effect of spin-dependent chemical pathways for transition metal SAC supported on graphene defects has been examined only recently. Orellana examined spin-constrained, non-equilibrium chemical pathways of $\mathrm{O}_{2}$ dissociation on transition metal SAC supported on a double vacancy $\mathrm{N}$ doped graphene defect and found small energy differences (below $0.1 \mathrm{eV}$ ) at points along these pathways increase the probability of spin-crossing during the reaction, enabling lower reaction barriers over spin-conserved reactions [29]. Our work expands this perspective to examine the magnetic moment of the surface defect and the TM and how this affects bonding energy. We then investigate the chemical activity during $\mathrm{CO}$ oxidation of four earth abundant $\mathrm{TM}$ atoms (V, Fe, Mo, Ta) coordinated with two graphene defect moieties using density functional theory (DFT). Single vacancy and pyridinic $\mathrm{N}$-doped single vacancy defects are two structurally similar graphene defect moieties that have similar charge transfer to metal adatoms but differing magnetic moments. $\mathrm{CO}$ oxidation is examined as it has many relevant industrial uses, including catalytic converters in vehicles, smoke stack emission control, and respiration filters [30].

Climbing image-nudged elastic band (CI-NEB) calculations of activation energy barriers of $\mathrm{CO}$ oxidation on all transition metal SAC in relaxed geometries on both defect moieties show all barrier energies on $\mathrm{N}$-doped single vacancy defects were $0.8 \mathrm{eV}$ or less. There were significant decreases in activation barrier energies for $\mathrm{V}$ and $\mathrm{Ta}$, no difference in the case of $\mathrm{Fe}$, and a slight increase for Mo when the $\mathrm{N}$ dopant was present. Hence, the common understanding of pyridinic $\mathrm{N}$ dopants improving catalytic behavior is simplistic. Due to the similar charge transfer calculated for both defect moieties, changes in activation barrier energy values were correlated with differences in bonding arrangements and local magnetic moment. A closer inspection of the density of states of the chemical pathways calculated via CI-NEB shows that lower activation energy barriers are associated with larger spin state asymmetry near the Fermi level in all cases, and in several cases the change in electronic charge transfer and bonding geometry was similar. Thus, spin state asymmetry provides new avenues for tailoring the molecular environment of promising support structures for SAC and guides choices for further experimental analysis, opening up additional lower energy chemical pathways for catalysis.

\subsection{Calculation Details}

Spin-polarized density functional theory calculations were implemented with the Vienna Ab initio Simulation Package (VASP) [31, 32] using the projector augmented wave (PAW) pseudopotentials $[33,34]$ and the generalized gradient approximation of the Perdew, Burke, and Ernzerhof (PBE) exchange-correlation functional [35], PBE incorporating a Hubbard correction term [36] to account for d electron self-interaction effects $(\mathrm{PBE}+\mathrm{U})$, as well as the strongly constrained and appropriately normed (SCAN) meta-generalized gradient approximation (meta-GGA) functional 
[37]. A Hubbard $\mathrm{U}$ of $2.0 \mathrm{eV}$ and an on-site exchange constant of $\mathrm{J}$ of $0 \mathrm{eV}$ were applied to the single Ta atom [38]. As the first meta-GGA, SCAN includes the gradient of the kinetic energy density, which satisfies 17 known possible exact constraints and can accurately capture intermediate range weak interactions in non-bonded systems and rare-gas atoms due to appropriate norming [37]. SCAN can often meet or even exceed the performance of more expensive hybrid calculations such as HSE06 (which were computationally intractable for the size of this system, with over 70 atoms) for many material properties [37, 39].

Binding energies, bond lengths, charge transfer, and magnetic moments were calculated for a Ta adatom bound to either a bare single vacancy (N0) or pyridinic N doped single vacancy $(\mathrm{N} 1)$ graphene substrate with $\mathrm{PBE}, \mathrm{PBE}+\mathrm{U}$, and SCAN, results listed in Table S1. All three functionals capture similar shifts in the Ta binding energy, charge transfer, and magnetic moments across the N0 and N1 defect moieties. However, the binding energies calculated by PBE are significantly closer in value to those calculated by SCAN than $\mathrm{PBE}+\mathrm{U}$. Additionally, the magnetic moments calculated by PBE are overall closer to the SCAN values than those calculated with $\mathrm{PBE}+\mathrm{U}$. To further verify the choice of the PBE functional, the total density of states (DOS) for Ta/N0 was calculated with PBE, PBE + U, and SCAN and plotted in Figure $\mathrm{S} 1$. The PBE and SCAN DOS plots both show a single spin down peak near the Fermi energy, while the PBE +U DOS shows two small spin down peaks. The DOS calculated with PBE is a closer match to the SCAN DOS than PBE $+\mathrm{U}$. Overall, comparison of the functionals demonstrate that $\mathrm{PBE}$ most accurately captured structural, electronic, and magnetic properties of interest for a single Ta atom secured to both graphene surface defect moieties investigated in this work.

The pseudopotential of each transition metal was chosen to represent the largest number of valence electrons for increased accuracy, while still utilizing the frozen core electron approximation for increased computational efficiency. In all calculations, the electron wave functions were expanded with a plane wave cut-off of $400 \mathrm{eV}$. The graphene substrates consist of a $6 \times 6$ repeating graphene unit cell with a vacuum spacing of $25 \AA$ between repeating images in the $\mathrm{z}$ direction. The large unit cell size and vacuum spacing were chosen to minimize spurious interactions between adsorbed adatoms due to the periodic boundary conditions.

For structural relaxations, reciprocal space was sampled with a $5 \times 5 \times 1$ gamma centered Monkhorst-Pack grid [40] and a Gaussian smearing of $0.01 \mathrm{eV}$. Standard convergence testing was performed with the reported cut-off and $\mathrm{k}$ point values chosen based on total energy values changing by less than $1 \mathrm{meV}$. The entire substrate was allowed to relax until the Hellmann-Feynman forces fell beneath $0.025 \mathrm{eV} / \AA$. The cohesive binding energies $\left(\mathrm{E}_{\mathrm{B}}\right)$ for each transition metal adatom were calculated via
$E_{B}=E_{G-T M}-E_{G}-E_{T M}$

$\mathrm{E}_{\mathrm{G}}$ represents the energy of the bare graphene substrate, $\mathrm{E}_{\mathrm{TM}}$ the energy of the isolated transition metal atom, and $\mathrm{E}_{\mathrm{G}-\mathrm{TM}}$ the total energy of the transition metal adatom adsorbed to the graphene substrate, each of which have been allowed to relax in identical hexagonal unit cells $(14.7 \times 14.7 \times 25 \AA)$. Charge density difference plots are defined by the following equation

$\Delta \mathrm{n}(\mathrm{r})=\mathrm{n}_{\mathrm{G}-\mathrm{TM}}-\mathrm{n}_{\mathrm{G}}-\mathrm{n}_{\mathrm{TM}}$

where $n_{G}$ represents the charge density of the optimized bare graphene substrate, $\mathrm{n}_{\mathrm{TM}}$ the charge density of the optimized transition metal adatom, and $\mathrm{n}_{\mathrm{G}-\mathrm{TM}}$ the charge density of the optimized total system of the transition metal adsorbed adatom to the graphene substrate.

Density of states calculations were performed with $9 \times 9 \times 1 \mathrm{k}$ points and a broader Gaussian smearing of $0.1 \mathrm{eV}$ for visualization. Charge transfer and transition state analysis were carried out with tools from the VASP Transition State Theory (VTST) package. Specifically, charge transfer was calculated with the Bader charge analysis method [41], as implemented by Henkelman et al. [42] Initial transition states along the minimum energy pathway were identified with the climbing image nudged elastic band method [43] and then further refined with the dimer method [44]. These two methods were also used to investigate spin-constrained reaction pathways of $\mathrm{CO}$ oxidation with the spin state specified using the NUPDOWN tag.

\section{Results and Discussion}

\subsection{Magnetic Properties of Graphene Defects}

The two common defect moieties in graphene that are investigated in this work have similar charge transfer to TM and differing magnetic moment $[45,46]$, as well as reported differences in activity of supported single atom catalysts such as $\mathrm{Pt}$ [44] and $\mathrm{Fe}$ [16]. These surfaces allow us to examine correlations between magnetic moment and reaction energy barriers. The first defect moiety is the single vacancy (NO) defect; the absence of the single carbon atom in the graphene lattice gives rise to a significant magnetic moment, with a calculated total value of $1.36 \mu_{\mathrm{B}}$, in agreement with previous reports $[47,48]$. The magnetization is mostly localized to a neighboring $\mathrm{C}$ atom from the vacancy (Fig. 1a). The second defect also consists of a single $\mathrm{C}$ vacancy with a neighboring $\mathrm{N}$-dopant in a pyridinic configuration (N1). Pyridinic $\mathrm{N}$ is a p-type doping site on graphene $[49,50]$ characterized as having two $\mathrm{C}$ bonds and known to contribute one electron to the delocalized graphene $\pi$ orbitals. The additional electron 

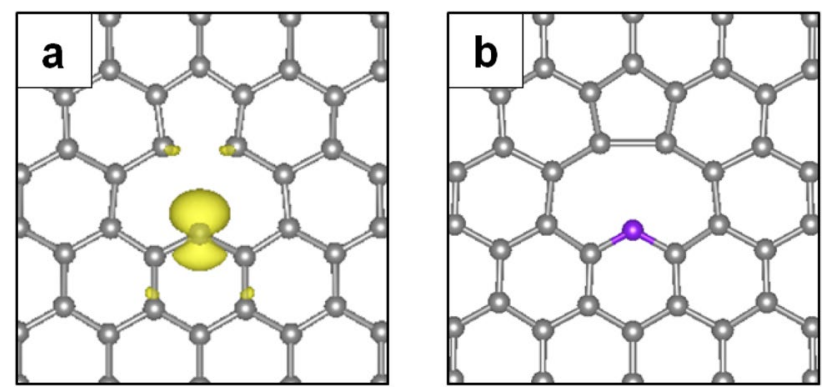

Fig. 1 Optimized geometry and magnetization density of a undoped single $\mathrm{C}$ vacancy (N0) and b single $\mathrm{C}$ vacancy with pyridinic $\mathrm{N}$ dopant (N1) on graphene surfaces. Grey atoms represent $\mathrm{C}$ while the purple atom is N. Isosurfaces drawn at a value of $0.01 \mathrm{e} / \AA^{3}$ to facilitate comparison with the literature $[47,51]$. N0 defect moiety has a total magnetic moment of $1.36 \mu_{\mathrm{B}}$ while $\mathrm{N} 1$ defect moiety has no magnetic moment

contributed by the pyridinic N-dopant extinguishes the magnetic moment of the N1 defect moiety (Fig. 1b). Additionally, the distance between the two remaining $\mathrm{C}$ nearest neighbor atoms shortens from 1.98 to $1.79 \AA$, forming a shorter bond with no magnetic moment.

Binding energies for all four transition metal (TM) atoms $(\mathrm{V}, \mathrm{Fe}, \mathrm{Mo}, \mathrm{Ta})$ on both defects in relaxed geometries are calculated and the electronic and magnetic properties are compared; binding energies, bond lengths, Bader charge transfer, and magnetic moments are summarized in Table S2. Pristine graphene is not analyzed, as both our previous work [17] and many examples in the literature have demonstrated that pristine graphene has unsuitably low adsorption and diffusion barrier energies to support single molecules or atoms [45, $52,53]$. Previous work on $\mathrm{N}$ defect moieties supported on graphene surfaces has associated enhanced catalytic activity due to the $\mathrm{N}$ dopants altering $\mathrm{O}_{2}$ binding energies with values approaching that of $\mathrm{O}_{2}$ on Pt surfaces [29, 54, 55]. However, the underlying mechanisms of this shift in binding energy is not completely understood. Here, the addition of the pyridinic $\mathrm{N}$ dopant in the $\mathrm{N} 1$ defect moiety consistently decreases the binding energy of the TM adatoms by an average value of $1.98 \mathrm{eV}$. The binding energy is plotted as a function of the charge transfer between the TM adatom and the surrounding defect, as calculated with Bader charge analysis, and is shown in Fig. 2a. There is remarkably little difference in the charge transferred between each TM atom when comparing N0 to N1. For example, in charge density difference plots of $\mathrm{Ta} / \mathrm{N} 0$ and $\mathrm{Ta} / \mathrm{N} 1$, shown, in Fig. $2 \mathrm{~b}$ and c, respectively, electronic charge is transferred from the adsorbed metal adatom to the surrounding graphene substrate and the two surfaces exhibit only minor differences in isosurface symmetry. All metal adatoms investigated become cationic independent of defect moiety. In contrast to the charge transfer, the binding energy and local magnetic
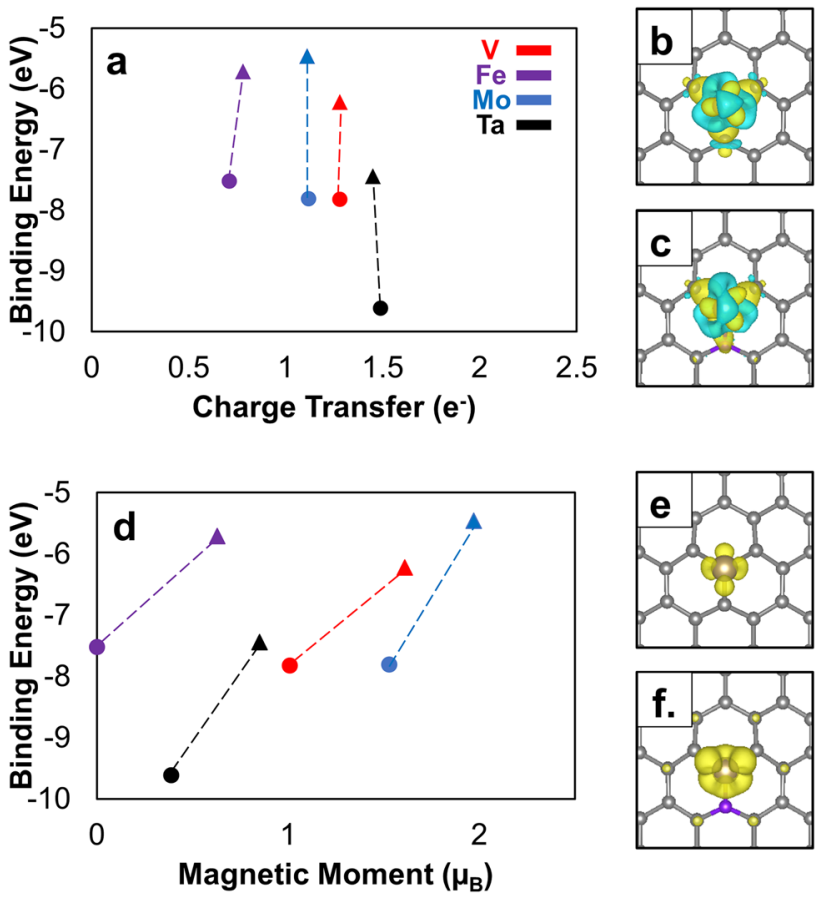

Fig. 2 The local a charge transfer and $\mathbf{d}$ magnetic moment plotted against the binding energy of the TM to N0 (circles) and N1 (triangles) defect moieties. Dashed lines are provided as guides for the eye. Charge density difference $\Delta \mathrm{n}(\mathrm{r})$ of $\mathbf{b} \mathrm{Ta} / \mathrm{N} 0$ and $\mathbf{c} \mathrm{Ta} / \mathrm{N} 1$. All isosurfaces are drawn at levels of $0.01 \mathrm{e} / \AA^{3}$. Yellow (blue) isosurfaces correspond to an increase (decrease) in charge density. Magnetization density isosurfaces of $\mathbf{e} \mathrm{Ta} / \mathrm{N} 0$ and $\mathbf{f} \mathrm{Ta} / \mathrm{N} 1$. Yellow isosurfaces correspond to spin up electron density. Grey and purple spheres correspond to carbon and nitrogen atoms, respectively

moment of the TM adatom both vary on the two defects as observed in Fig. 2d. Plots of the total magnetic moment illustrate the strong localization of the magnetic moment while also illustrating the increase in magnetization from the $\mathrm{N} 0$ to the N1 defect moiety, and is shown for the case of Ta in Fig. 2e and f, respectively. (Charge density difference and magnetization plots for all TM atoms can be found in Figure S2 of the Supplementary Information.) With the adsorption of a TM atom, the total magnetization of the N1 system increases by an average of $0.92 \mu_{\mathrm{B}}$, approaching the value of one additional unpaired electron. Thus, these graphene defect moieties allow one to assess the effect of magnetic moment nearly independent from charge transfer. Furthermore, the bonding geometry of metal adatoms on both defect moieties does not significantly change as shown in Table S2. The trend of defects with higher magnetic moments inducing stronger binding energies was also identified for $\mathrm{Fe}$ and other TM on similar N-doped graphene defects [29, 46]. Kattel et al. found that when the final coordinated TM SAC exhibited a magnetic moment, the binding energy became weaker [56], similar to our results for TM/N1, which have higher magnetic moments in the final state geometry and 
weaker binding energies. Considering that previous work has correlated the binding energy of $\mathrm{O}_{2}$ with enhanced catalytic activity, this suggests that tuning the magnetic moment of an impurity on a defect site provides an additional knob to optimize catalytic performance.

\subsection{Effect of Magnetic Moment on Gas Adsorption and $\mathrm{CO}$ Oxidation}

The binding energies of $\mathrm{CO}, \mathrm{CO}_{2}$, and $\mathrm{O}_{2}$ molecules, involved in CO oxidation, were calculated for both $\mathrm{N} 0$ and $\mathrm{N} 1 \mathrm{SAC}$ systems to assess resistance to $\mathrm{CO}$ poisoning and elucidate chemical pathways. The binding energy of $\mathrm{CO}_{2}$ is weak and in some cases positive; the values are shown in Table S3 of Supporting Information. $\mathrm{O}_{2}$ is always adsorbed more strongly than $\mathrm{CO}$, which is favorable to mitigate $\mathrm{CO}$ poisoning of the catalyst [57] on all metal adatoms investigated on both defect moieties. Figure 3a plots the binding energy trends for $\mathrm{O}_{2}$ and $\mathrm{CO}$ and illustrates that gas molecules generally adsorb more strongly to TM SAC coordinated to N1, all of which were shown to have higher magnetic moments compared to N0 (Table S2). All gas molecule magnetic moment values are tabulated in Table S3 of the Supporting Information. This is consistent with data shown in Fig. 2d where bare N0 has a higher magnetic moment and consequently higher $\mathrm{E}_{\mathrm{B}}$ to $\mathrm{SAC}$ than bare $\mathrm{N} 1$. One notable exception is $\mathrm{O}_{2}-\mathrm{V}$, where the magnetic moment on the $\mathrm{O}_{2}-\mathrm{V}$ system is calculated as zero on both defect types. The total density of states (DOS) for each TM SAC was also calculated and shown in Figure S4. Correlation between gas molecule

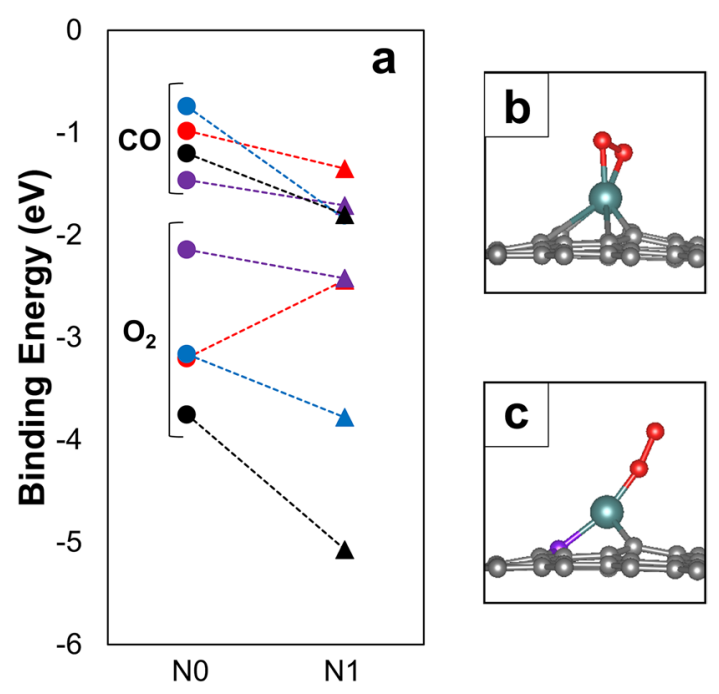

Fig. 3 a The binding energy of $\mathrm{CO}$ and $\mathrm{O}_{2}$ adsorbed to each TM on $\mathrm{NO}$ (circles) and N1 (triangles) defect moieties for Fe (purple), V (red), Mo (blue), and Ta (black). Relaxed bonding geometry of $\mathrm{O}_{2}$ adsorbed to $\mathbf{b} \mathrm{V} / \mathrm{N} 0$, note the side-on configuration of the linear $\mathrm{O}_{2}$ molecule, $\mathbf{c}$ V/N1 where the $\mathrm{O}_{2}$ molecule adsorbs in an end-on configuration. Grey, purple, red, and green spheres correspond to carbon, nitrogen, oxygen, and vanadium atoms, respectively

binding energy with proximity of d-orbitals to the Fermi level was not observed by comparing DOS in Figure S4 and calculated binding energies of gas molecules. For example, the DOS of Fe/N0 and V/N1 would predict higher gas binding energies than $\mathrm{Fe} / \mathrm{N} 1$ and $\mathrm{V} / \mathrm{N} 0$, respectively, by this criterion. However, both $\mathrm{O}_{2}$ and $\mathrm{CO}$ have higher binding energies on $\mathrm{Fe} / \mathrm{N} 1$ versus $\mathrm{Fe} / \mathrm{N} 0$ as shown in Fig. 3. Similarly, the $\mathrm{CO}$ binding energy increases on V/N1 with respect to V/N0, while on the other hand, the $\mathrm{O}_{2}$ binding energy is much weaker on V/N1. Interestingly, a shift from side-on (Fig. 3b) for the N0 defect to end-on (Fig. 3c) for the N1 defect bonding geometry is observed. Here we postulate that the additional unpaired electron contributed by the N1 defect favors the end-on configuration for $\mathrm{O}_{2}-\mathrm{V}$ to obtain a ground state with no magnetic moment and lower system energy. Bonding geometry changes have been associated with minimizing magnetic moment to lower system energy in a few other studies [29,58]. This result would explain the weaker binding energy for $\mathrm{O}_{2}-\mathrm{V}$ on $\mathrm{N} 1$, as the end-on geometry appears to minimize the additional magnetic moment imparted by the $\mathrm{N}$ dopant. In line with previous work that correlates the binding energy of $\mathrm{O}_{2}$ to catalytic performance [54, 59, 60], we are motivated to examine more closely the effect of binding energy and magnetic moment on $\mathrm{CO}$ oxidation barrier energy.

The relatively weaker binding energy of $\mathrm{CO}$ versus $\mathrm{O}_{2}$ in Fig. 3a suggest that the modified Eley-Rideal reaction [18, 61] pathway (wherein $\mathrm{CO}$ reacts with adsorbed $\mathrm{O}_{2}$ to form $\mathrm{CO}_{2}$ and $\mathrm{O}$ ) is most appropriate for this system, rather than the Langmuir-Hinshelwood reaction mechanism. Fe/NO has previously been found to proceed via the modified Eley-Rideal pathway as well [62]. The climbing image nudged elastic band (CI-NEB) method was used to model the modified Eley-Rideal reaction path and calculate the barrier energies without constraining the spin state, typically done in prior studies, for each combination of a TM-SAC and the graphene defect moieties investigated. Previous work investigating spinconstrained reaction pathways revealed that these pathways are close in energy for TM SACs on graphene defects, increasing the likelihood of spin inversion events between reaction steps that benefit catalytic activity $[29,63]$, making spin unrestricted calculations more appropriate for these systems.

The calculated barrier energies between the initial state (IS) and transition state (TS), listed in Table 1, were under $1 \mathrm{eV}$ and impressively, all barrier energies for $\mathrm{N} 1$ were less than $0.8 \mathrm{eV}$. Generally, reactions below $0.8 \mathrm{eV}$ are considered feasible at room temperature [64]. All relaxed geometries and energy levels of IS, TS, and final states (FS) for each TM are

Table 1 Activation barrier energies of $\mathrm{CO}$ oxidation on different defect moieties

\begin{tabular}{lllll}
\hline & V & Fe & Mo & Ta \\
\hline N0 & 0.98 & 0.74 & 0.76 & 0.91 \\
N1 & 0.55 & 0.74 & 0.83 & 0.66 \\
\hline
\end{tabular}


provided in the Supplementary Information in Figure S3, S4, and Table S4. The energy barrier calculated for Fe/N0 is in agreement with previous reports $[62,63]$. For all TM SAC on $\mathrm{N} 1$, the addition of a pyridinic $\mathrm{N}$ dopant appears to favor the formation of a $\mathrm{COOO}$ intermediate [65-67] at the transition state, with the exception of Ta (see Figure S6). On N0, Fe SAC also forms a similar intermediate and all others do not (see Figure S5). Interestingly, as observed in Table 1, the Fe SAC barrier energies are the same on both defect moieties despite $\mathrm{N}$ doped defects often being attributed to increasing catalytic activity for both Fe [54] and Pt [68] SAC. Overall, for all SAC on N1 and N0 defect moieties, there is no clear trend of the relationship with defect moiety and energy barrier. V and Ta both demonstrated significant decreases in activation barrier energies, Fe had no change, and Mo showed a slight increase in value on $\mathrm{N} 1$ with respect to N0. Insight of physical mechanisms behind these trends can be obtained by examining the spin polarized projected density of states (PDOS) of the transition states, shown in Figs. 4, 5 and 6. We find that the magnetic state of atomic and molecular orbitals near the Fermi level exhibits common features, i.e., asymmetry of spin states, for systems with lowest activation energy barriers. Each TM SAC will be discussed in the following section in turn.

The relaxed geometries and energy levels for $\mathrm{CO}$ oxidation on Mo on the two defect types are illustrated in Fig. 4a. The barrier energy is approximately $9 \%$ higher on N1 with respect to N0, where values are shown in Table 1. We systematically examine physical mechanisms, starting with geometry, charge transfer and induced magnetic moment as past studies clearly indicate geometry and charge transfer play a role and we seek to find cases where only magnetic moment changes. The geometry of $\mathrm{O}_{2}$ is the same, side-on, for both moieties, illustrated by the insets of Fig. 4a. Additionally, charge transfer and even magnetic moment of $\mathrm{O}_{2}$ on Mo/N0 and Mo/N1 TS are very similar, values are shown in Table S4. The main observable difference is the PDOS. In Fig. 4c, Mo/N1 shows spin state asymmetry in the $\mathrm{O}_{2}$ peaks near the Fermi level, however the spin state splitting of these peaks is more pronounced for Mo/N0 (Fig. 4b). Additionally, near the Fermi level (within $1 \mathrm{eV}$ ), Mo/N1 shows greater overlap between $\mathrm{CO}, \mathrm{O}_{2}$, and Mo 4d orbital peaks, however Mo/N0 retains a slightly lower energy barrier, emphasizing the unique effect of spin split orbitals on reactivity. Spin state splitting of the orbital peaks with the greatest proximity to the Fermi level was also present in the DOS of the best performing Au SAC analyzed in a previous theoretical work [69]. Furthermore, the PDOS of $\mathrm{O}_{2}-\mathrm{Fe}$ on both defect moieties, shown in Figure S5, has the same CO oxidation energy barrier for both $\mathrm{Fe} / \mathrm{N} 0$ and Fe/N1. This can be understood by considering the similar level of spin state asymmetry near the Fermi level on both defects. Even though there is greater charge transfer to $\mathrm{O}_{2}$ adsorbed to $\mathrm{Fe} /$ $\mathrm{N} 1$, and a greater local magnetic moment for $\mathrm{O}_{2}$ adsorbed to $\mathrm{Fe} / \mathrm{N} 0$, the energy barrier values most closely correlate to the behavior of the spin split orbitals near the Fermi level in the PDOS.

The chemical activity of Ta SAC for $\mathrm{CO}$ oxidation showed a significant improvement on $\mathrm{N} 1$ in terms of lower barrier energy compared to $\mathrm{Ta} / \mathrm{N} 0$, with an activation energy barrier of $0.66 \mathrm{eV}$ compared to $0.91 \mathrm{eV}$ as shown in Fig. 5a. The electronic charge transfer to $\mathrm{O}_{2}$, shown in Table $\mathrm{S} 4$, for $\mathrm{Ta} / \mathrm{N} 0$ and $\mathrm{Ta} / \mathrm{N} 1$ was remarkably similar. Furthermore, the geometric structures of $\mathrm{O}_{2}$ on the $\mathrm{Ta} / \mathrm{N} 0$ and Ta/N1 TS, observed in the insets of Fig. 5a,
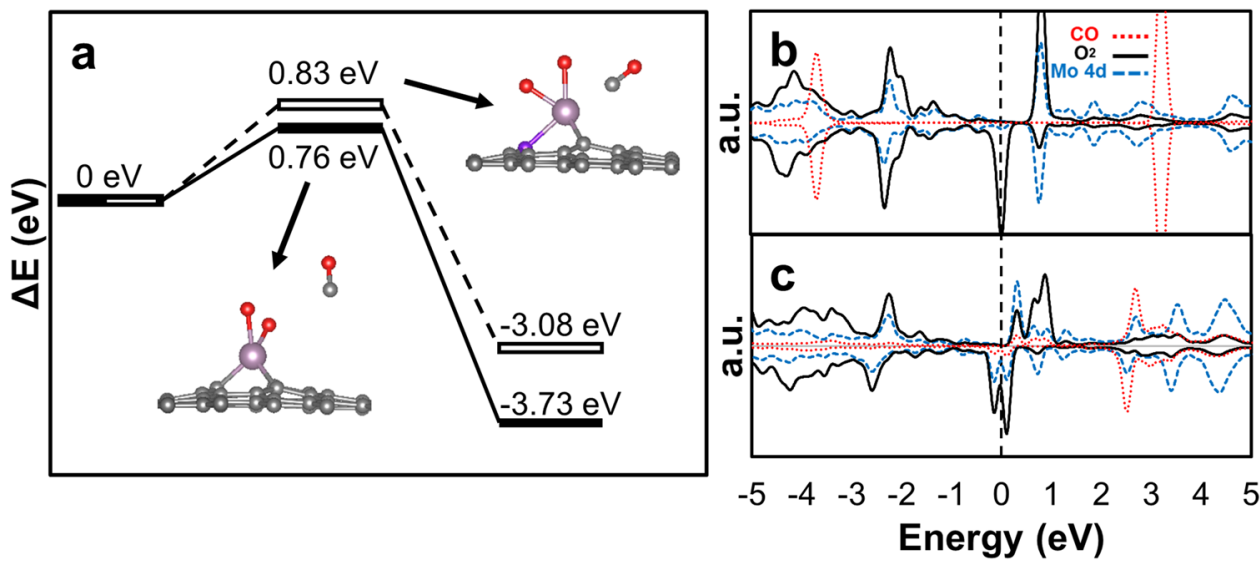

Fig. 4 a Energy levels of the initial state (IS), transition state (TS), and final state (FS) for N0 (solid black) and N1 (hollow bar). Insets show relaxed geometry for TS of $\mathrm{O}_{2}-\mathrm{Mo} / \mathrm{N} 0$ and $\mathrm{O}_{2}-\mathrm{Mo} / \mathrm{N} 1$. Grey, purple, red, and light purple spheres correspond to $\mathrm{C}, \mathrm{N}, \mathrm{O}$, and Mo atoms, respectively. Spin polarized projected density of states (PDOS) for TS of $\mathbf{b} \mathrm{O}_{2}-\mathrm{Mo} / \mathrm{N0}$ and $\mathbf{c} \mathrm{O}_{2}-\mathrm{Mo} / \mathrm{N} 1$ with Fermi energy

highlighted with a vertical dashed line. Blue dashed curve shows local PDOS of adsorbed TM adatom d orbitals. Red dotted and black solid curves show local PDOS for $\mathrm{CO}$ and $\mathrm{O}_{2}$ molecules, respectively. All positive values correspond to spin up and all negative values are spin down 

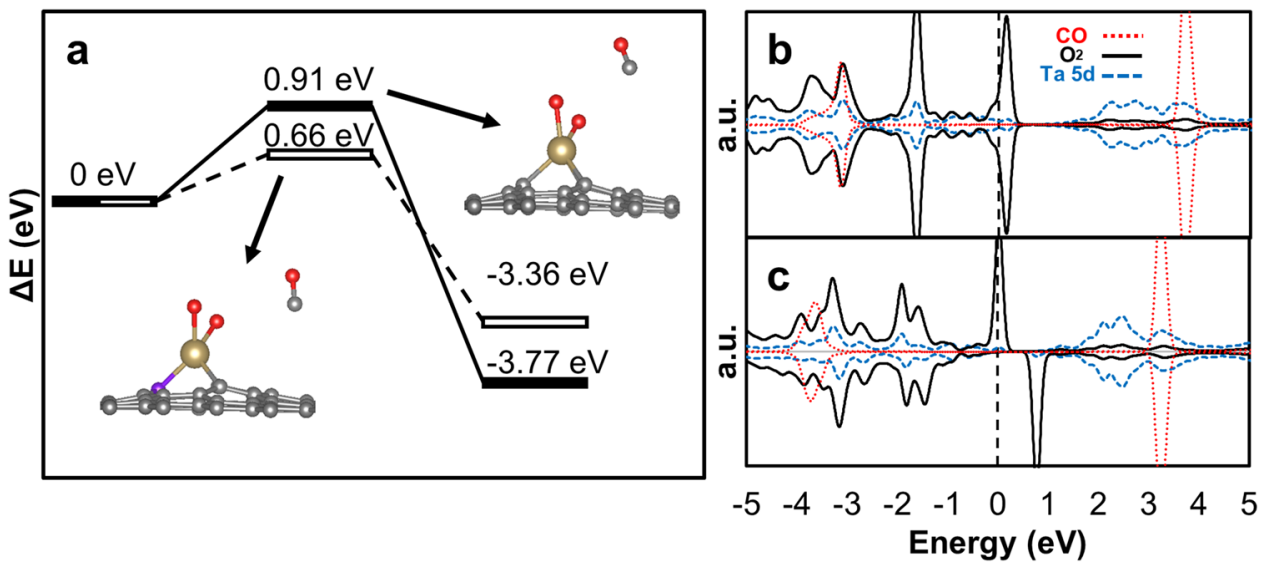

Fig. 5 a Energy levels of the initial state (IS), transition state (TS), and final state (FS) for N0 (solid black) and N1 (hollow bar). Insets show relaxed geometry for TS of $\mathrm{O}_{2}-\mathrm{Ta} / \mathrm{N} 0$ and $\mathrm{O}_{2}-\mathrm{Ta} / \mathrm{N} 1$. Grey, purple, red, and yellow spheres correspond to $\mathrm{C}, \mathrm{N}, \mathrm{O}$, and Ta atoms, respectively. Spin polarized density of states for TS of $\mathbf{b} \mathrm{O}_{2}-\mathrm{Ta} / \mathrm{N} 0$ and $\mathbf{c} \mathrm{O}_{2}-\mathrm{Ta} / \mathrm{N} 1$. Blue dashed curve shows local PDOS of adsorbed

both exhibit a side-on bonding configuration (see also Table S2). In contrast to the similar charge transfer and geometry between Ta defect moieties, there is a clear difference in the PDOS of Ta/N0 compared to Ta/N1. The symmetry of the PDOS of Ta/N0, in Fig. 5b, has no discernable magnetic moment and shows a higher number of states very close to the Fermi level (within $1 \mathrm{eV}$ ). Additionally, both the PDOS of Ta/N0 and Ta/N1 have similar densities of Ta $5 \mathrm{~d}$ orbitals near the Fermi level. However, the PDOS for the Ta/N1 TS (Fig. 5c) shows a large spin state asymmetry near the Fermi level for the adsorbed $\mathrm{O}_{2}$ molecule. Thus, spin state asymmetry appears to destabilize the $\mathrm{O}_{2}$ bond, increasing reactivity and lowering the barrier energy for $\mathrm{CO}$ oxidation on $\mathrm{Ta} / \mathrm{N} 1$ by $27 \%$.

The initial, transition, and final states of $\mathrm{CO}$ oxidation on $\mathrm{Ta} / \mathrm{N} 0$ produced with NEB calculations had zero magnetic moment. This motivated additional NEB calculations to compare the activation barrier energies of spinconstrained reaction pathways having differing spin states. By constraining the magnetic moment of the $\mathrm{CO}$ oxidation reaction facilitated by $\mathrm{Ta} / \mathrm{N} 0$ to be either 1 (doublet) or 3 (quartet) states, we found significantly lower energy barriers compared to the non-magnetic spin state, shown in Table 2. The energy barriers of the doublet and quartet spin states were found to be quite similar with less than $0.05 \mathrm{eV}$ of difference. Since mainly the frontier orbital affects bonding, magnetic moment alone does not explain differences in chemical activity. Higher spin states may consist of unpaired electrons in both the frontier as well as orbitals further away from the Fermi energy, thus they are expected to have similar energy barriers when the spin asymmetry near the Fermi energy is similar.

TM adatom d orbitals. Red dotted and black solid curves show local PDOS for $\mathrm{CO}$ and $\mathrm{O}_{2}$ molecules, respectively. The Fermi levels have been set to $0 \mathrm{eV}$, indicated with a vertical dashed black line. All positive values correspond to spin up and all negative values are spin down

Table 2 Activation barrier energies of spin-constrained $\mathrm{CO}$ oxidation on $\mathrm{Ta} / \mathrm{N} 0$

\begin{tabular}{lll}
\hline Defect moiety & $\mathrm{E}_{\mathrm{A}}[\mathrm{eV}]$ \\
\hline Ta/N0 & $\mathrm{m}=0$ & 0.91 \\
& $\mathrm{~m}=1$ & 0.36 \\
& $\mathrm{~m}=3$ & 0.32 \\
\hline
\end{tabular}

Lastly, we consider the impressive improvement between $\mathrm{V} / \mathrm{N} 0$ and $\mathrm{V} / \mathrm{N} 1$, a decrease in activation energy barrier of $44 \%$, comparable to CO oxidation on a Pt surface [70]. The adsorbing $\mathrm{O}_{2}$ molecule undergoes a shift in geometry, from side-on to end-on absorption for the N0 and N1 molecular environments, respectively, as discussed previously and which can be seen in the insets of Fig. 6a. This shift is not thought to result in a beneficial steric advantage. Despite the the increased proximity of the end-on $\mathrm{O}_{2}$ molecule to the reacting physisorbed $\mathrm{CO}$ molecule, previous work has found that a similar end-on $\mathrm{O}_{2}$ adsorbed on Au SAC displayed significantly higher activation energy barriers for $\mathrm{CO}$ oxidation than side-on [69]. The increased magnetic moment conferred by the $\mathrm{N} 1$ defect moiety could also be responsible for the change in $\mathrm{O}_{2}$ adsorption geometry from side-on to end-on, which agrees with a finding demonstrated by Orellana for a similar system [29]. Rather than steric advantage explaining the lower energy barrier for $\mathrm{V} / \mathrm{N} 1$, there is a strong spin state asymmetry in the $\mathrm{V}$ peaks near the Fermi level, larger in energy separation and magnitude as compared to the TS for V/N0 (Fig. 6b). This effect could explain why the activation energy barrier for $\mathrm{V} / \mathrm{N} 1$ is $44 \%$ lower than $\mathrm{V} / \mathrm{N} 0$, despite $\mathrm{V} /$ N0 having more charge transfer to $\mathrm{O}_{2}$ shown in Table S4. Thus spin state asymmetry appears to lower the barrier energies for $\mathrm{CO}$ oxidation for all systems. 

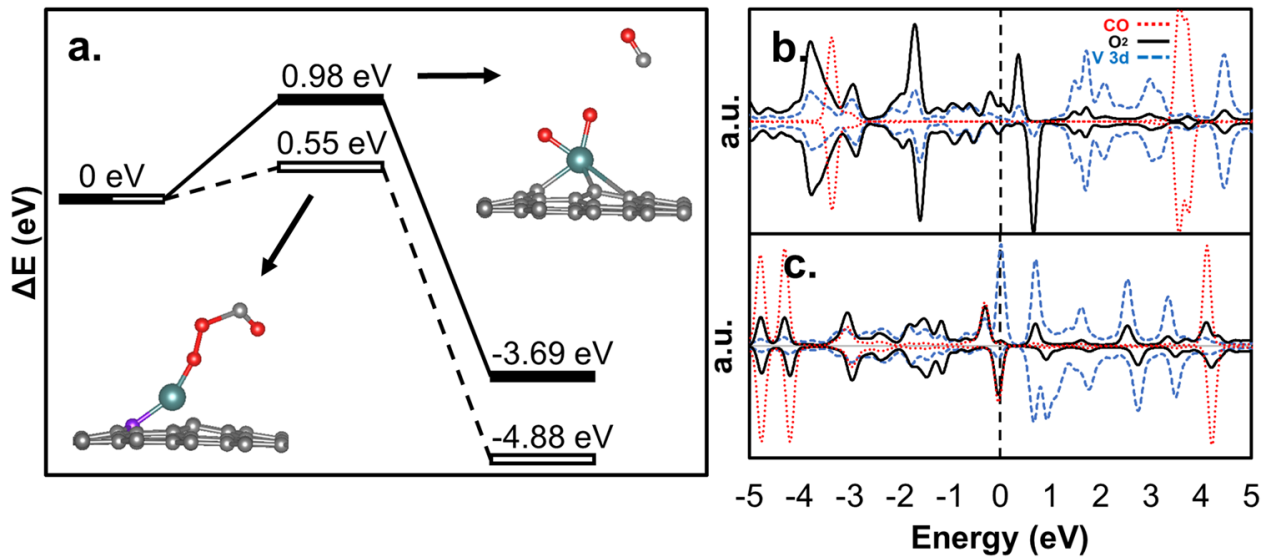

Fig. 6 a Energy levels of the initial state (IS), transition state (TS), and final state (FS) for N0 (solid black) and N1 (hollow bar). Insets show relaxed geometry for TS of $\mathrm{O}_{2}-\mathrm{V} / \mathrm{NO}$ and $\mathrm{O}_{2}-\mathrm{V} / \mathrm{N} 1$. Grey, purple, red, and green spheres correspond to $\mathrm{C}, \mathrm{N}, \mathrm{O}$, and $\mathrm{V}$ atoms, respectively. Spin polarized density of states for TS of $\mathbf{b} \mathrm{O}_{2}-\mathrm{V} / \mathrm{N} 0$ and $\mathbf{c} \mathrm{O}_{2}-\mathrm{V} / \mathrm{N} 1$. Blue dashed curve shows local PDOS of adsorbed

\section{Conclusion}

Through first principles calculations, the performance of a wide range of earth-abundant transition metals ( $\mathrm{V}, \mathrm{Fe}, \mathrm{Mo}$, and $\mathrm{Ta}$ ) as $\mathrm{SAC}$ for $\mathrm{CO}$ oxidation on two types of graphene surface defects (N0, N1) has been evaluated. Further, by calculating the activation energy barriers of $\mathrm{CO}$ oxidation reactions facilitated by these stabilized SAC through a modified Eley-Rideal pathway, we found that all four metals have energy barriers $<1 \mathrm{eV}$ on both defect types and significantly the pyridinic $\mathrm{N}$ dopant accesses energy barriers below $0.8 \mathrm{eV}$ for all metals. Compared to our previous work modelling the same reaction with single atom Pt for a Langmuir-Hinshelwood pathway, V on N1 is comparable to the performance of a Pt surface [70] and is the most competitive with SAC Pt catalysts $[17,71]$ with an activation energy of $0.55 \mathrm{eV}$. The spin polarized projected density of states shows that greater spin state asymmetry in atomic and molecular orbital peaks near the Fermi level is associated with lower activation energy barriers, where associated decreases in activation barrier energies of $\mathrm{CO}$ oxidation of $44 \%$ and $27 \%$ were observed for $\mathrm{V}$ and $\mathrm{Ta}$, respectively. Our work both extends the range of earth-abundant choices for SAC and reveals the importance of molecular coordination affecting the magnetic moment and in turn binding energies and activation energy barriers. This provides further means for improving the chemical activity of earth-abundant transition metals. Parsing the large parameter space composed of TM elements and surface defects will aid in the design of earth abundant and sustainable catalysts. These results can guide selection of materials for investigations using techniques such as high-angle annular dark-field-scanning
TM adatom d orbitals. Red dotted and black solid curves show local PDOS for $\mathrm{CO}$ and $\mathrm{O}_{2}$ molecules, respectively. The Fermi levels have been set to $0 \mathrm{eV}$, indicated with a vertical dashed black line. All positive values correspond to spin up and all negative values are spin down

transmission electron microscopy (HAADF-STEM) and spin-resolved electron energy loss spectroscopy (EELS) to probe chemical bonding and local electronic structure alongside with in situ catalytic reactions. Relationships between molecular coordination, local magnetic moment, and catalytic activity gleaned from experiments can further elucidate how spin state can provide an additional knob for tuning chemical activity. A promising future avenue is to explore the effect of spin on other molecular coordinations of TM SAC on graphene in order to identify systems that may meet or exceed the performance of PGM.

Supplementary Information The online version contains supplementary material available at https://doi.org/10.1007/s10562-021-03737-y.

Acknowledgements This work used the Extreme Science and Engineering Discovery Environment (XSEDE), which is supported by National Science Foundation Grant No. ACI-1548562. The XSEDE allocation account used for this research was TG-DMR180078. This research was partially supported by the National Science Foundation Materials Research Science and Engineering Center program through the UC Irvine Center for Complex and Active Materials (DMR2011967). Additional support was provided by the National Science Foundation Graduate Research Fellowship under Grant No. DGE1839285 and the XSEDE EMPOWER program.

Open Access This article is licensed under a Creative Commons Attribution 4.0 International License, which permits use, sharing, adaptation, distribution and reproduction in any medium or format, as long as you give appropriate credit to the original author(s) and the source, provide a link to the Creative Commons licence, and indicate if changes were made. The images or other third party material in this article are included in the article's Creative Commons licence, unless indicated otherwise in a credit line to the material. If material is not included in the article's Creative Commons licence and your intended use is not permitted by statutory regulation or exceeds the permitted use, you will 
need to obtain permission directly from the copyright holder. To view a copy of this licence, visit http://creativecommons.org/licenses/by/4.0/.

\section{References}

1. Bai J, Zhu Q, Lv Z, Dong H, Yu J, Dong L (2013) Nitrogen-doped graphene as catalysts and catalyst supports for oxygen reduction in both acidic and alkaline solutions. Int J Hydrogen Energy 38(3):1413-1418. https://doi.org/10.1016/j.ijhydene.2012.11.039

2. Qu L, Liu Y, Baek J-B, Dai L (2010) Nitrogen-doped graphene as efficient metal-free electrocatalyst for oxygen reduction in fuel cells. ACS Nano 4(3):1321-1326. https://doi.org/10.1021/nn901 $850 \mathrm{u}$

3. Gasteiger HA, Kocha SS, Sompalli B, Wagner FT (2005) Activity benchmarks and requirements for Pt, Pt-alloy, and non-Pt oxygen reduction catalysts for PEMFCs. Appl Catal B 56(1):9-35. https:// doi.org/10.1016/j.apcatb.2004.06.021

4. Cheng N, Stambula S, Wang D, Banis MN, Liu J, Riese A, Xiao B, Li R, Sham T-K, Liu L-M, Botton GA, Sun X (2016) Platinum single-atom and cluster catalysis of the hydrogen evolution reaction. Nat Commun 7(1):13638. https://doi.org/10.1038/ncomm s13638

5. Rivera-Cárcamo C, Serp P (2018) Single atom catalysts on carbon-based materials. ChemCatChem 10(22):5058-5091. https:// doi.org/10.1002/cctc.201801174

6. Yoo E, Okata T, Akita T, Kohyama M, Nakamura J, Honma I (2009) Enhanced electrocatalytic activity of pt subnanoclusters on graphene nanosheet surface. Nano Lett 9(6):2255-2259. https:// doi.org/10.1021/n1900397t

7. Jiang K, Back S, Akey AJ, Xia C, Hu Y, Liang W, Schaak D, Stavitski E, Nørskov JK, Siahrostami S, Wang H (2019) Highly selective oxygen reduction to hydrogen peroxide on transition metal single atom coordination. Nat Commun 10(1):3997. https:// doi.org/10.1038/s41467-019-11992-2

8. Yang S, Tak YJ, Kim J, Soon A, Lee H (2017) Support effects in single-atom platinum catalysts for electrochemical oxygen reduction. ACS Catal 7(2):1301-1307. https://doi.org/10.1021/acscatal. $6 \mathrm{~b} 02899$

9. Wang A, Li J, Zhang T (2018) Heterogeneous single-atom catalysis. Nat Rev Chem 2(6):65-81. https://doi.org/10.1038/ s41570-018-0010-1

10. Lu J, Serna P, Aydin C, Browning ND, Gates BC (2011) Supported molecular iridium catalysts: resolving effects of metal nuclearity and supports as ligands. J Am Chem Soc 133(40):16186-16195. https://doi.org/10.1021/ja206486j

11. Getsoian A, Das U, Camacho-Bunquin J, Zhang G, Gallagher JR, Hu B, Cheah S, Schaidle JA, Ruddy DA, Hensley JE, Krause TR, Curtiss LA, Miller JT, Hock AS (2016) Organometallic model complexes elucidate the active gallium species in alkane dehydrogenation catalysts based on ligand effects in Ga K-edge XANES. Catal Sci Technol 6(16):6339-6353. https://doi.org/10.1039/ C6CY00698A

12. Qiao B, Wang A, Yang X, Allard LF, Jiang Z, Cui Y, Liu J, Li J, Zhang T (2011) Single-atom catalysis of CO oxidation using Pt1/ FeOx. Nature Chem 3(8):634-641. https://doi.org/10.1038/nchem. 1095

13. Yang X-F, Wang A, Qiao B, Li J, Liu J, Zhang T (2013) Singleatom catalysts: a new frontier in heterogeneous catalysis. Acc Chem Res 46(8):1740-1748. https://doi.org/10.1021/ar300361m

14. Yan D, Li Y, Huo J, Chen R, Dai L, Wang S (2017) Defect chemistry of nonprecious-metal electrocatalysts for oxygen reactions. Adv Mater 29(48):1606459. https://doi.org/10.1002/adma.20160 6459
15. Li L, Chang X, Lin X, Zhao Z-J, Gong J (2020) Theoretical insights into single-atom catalysts. Chem Soc Rev 49(22):81568178. https://doi.org/10.1039/D0CS00795A

16. Zhang H, Li J, Xi S, Du Y, Hai X, Wang J, Xu H, Wu G, Zhang J, Lu J, Wang J (2019) A graphene-supported single-atom $\mathrm{FeN}_{5}$ catalytic site for efficient electrochemical $\mathrm{CO}_{2}$ reduction. Angew Chem Int Ed 58(42):14871-14876. https://doi.org/10.1002/anie. 201906079

17. Wang CS, Wang H, Wu R, Ragan R (2018) Evaluating the stability of single-atom catalysts with high chemical activity. J Phys Chem C 122(38):21919-21926. https://doi.org/10.1021/acs.jpcc.8b066 21

18. Esrafili MD, Heydari S (2018) CO oxidation catalyzed by a single Ti atom supported on divacancy defective graphene: a dispersioncorrected DFT study. ChemistrySelect 3(16):4471-4479. https:// doi.org/10.1002/slct.201800573

19. Song M, Song Y, Sha W, Xu B, Guo J, Wu Y (2020) Recent advances in non-precious transition metal/nitrogen-doped carbon for oxygen reduction electrocatalysts in PEMFCs. Catalysts 10(1):141. https://doi.org/10.3390/catal10010141

20. Seabourne CR, Scott AJ, Brydson R, Nicholls RJ (2009) A systematic approach to choosing parameters for modelling fine structure in electron energy-loss spectroscopy. Ultramicroscopy 109(11):1374-1388. https://doi.org/10.1016/j.ultramic.2009.07. 002

21. Idrobo JC, Rusz J, Spiegelberg J, McGuire MA, Symons CT, Vatsavai RR, Cantoni C, Lupini AR (2016) Detecting magnetic ordering with atomic size electron probes. Adv Struct Chem Imag 2(1):5. https://doi.org/10.1186/s40679-016-0019-9

22. Negi D, Zeiger PM, Jones L, Idrobo J-C, van Aken PA, Rusz J (2019) Prospect for detecting magnetism of a single impurity atom using electron magnetic chiral dichroism. Phys Rev B 100(10):104434. https://doi.org/10.1103/PhysRevB.100.104434

23. Witt JA, Mumm DR, Mohraz A (2016) Microstructural tunability of co-continuous bijel-derived electrodes to provide high energy and power densities. J Mater Chem A 4(3):1000-1007. https://doi. org/10.1039/C5TA06260H

24. Garcia AE, Wang CS, Sanderson RN, McDevitt KM, Zhang Y, Valdevit L, Mumm DR, Mohraz A, Ragan R (2019) Scalable synthesis of gyroid-inspired freestanding three-dimensional graphene architectures. Nanoscale Adv 1(10):3870-3882. https://doi.org/10. 1039/C9NA00358D

25. Torquato S, Hyun S, Donev A (2002) Multifunctional composites: optimizing microstructures for simultaneous transport of heat and electricity. Phys Rev Lett 89(26):266601. https://doi.org/10.1103/ PhysRevLett.89.266601

26. Chen H-Y, Kwon Y, Thornton K (2009) Multifunctionality of three-dimensional self-assembled composite structure. Scripta Mater 61(1):52-55. https://doi.org/10.1016/j.scriptamat.2009. 03.006

27. Yang B, Gagliardi L, Truhlar DG (2018) Transition states of spinforbidden reactions. Phys Chem Chem Phys 20(6):4129-4136. https://doi.org/10.1039/C7CP07227A

28. Pichugina DA, Polynskaya YG, Kuz'menko NE (2016) Spin and structural features of oxygen dissociation on tetrahedral $\mathrm{Ag}_{20}$ and $\mathrm{Ag}_{19}$ Au clusters. Phys Chem Chem Phys 18(27):18033-18044. https://doi.org/10.1039/C6CP01630H

29. Orellana W (2013) Catalytic properties of transition metal- $\mathrm{N}_{4}$ moieties in graphene for the oxygen reduction reaction: evidence of spin-dependent mechanisms. J Phys Chem C 117(19):98129818. https://doi.org/10.1021/jp4002115

30. Soliman NK (2019) Factors affecting CO oxidation reaction over nanosized materials: a review. J Market Res 8(2):2395-2407. https://doi.org/10.1016/j.jmrt.2018.12.012

31. Kresse G, Furthmüller J (1996) Efficiency of Ab-initio total energy calculations for metals and semiconductors using a plane-wave 
basis set. Comput Mater Sci 6(1):15-50. https://doi.org/10.1016/ 0927-0256(96)00008-0

32. Kresse G, Furthmüller J (1996) Efficient iterative schemes for Ab initio total-energy calculations using a plane-wave basis set. Phys Rev B 54(16):11169-11186. https://doi.org/10.1103/PhysR evB.54.11169

33. Kresse G, Joubert D (1999) From ultrasoft pseudopotentials to the projector augmented-wave method. Phys Rev B 59(3):1758-1775. https://doi.org/10.1103/PhysRevB.59.1758

34. Blöchl PE (1994) Projector augmented-wave method. Phys Rev B 50(24):17953-17979. https://doi.org/10.1103/PhysRevB.50. 17953

35. Perdew JP, Burke K, Ernzerhof M (1996) Generalized gradient approximation made simple. Phys Rev Lett 77(18):3865-3868. https://doi.org/10.1103/PhysRevLett.77.3865

36. Dudarev SL, Botton GA, Savrasov SY, Humphreys CJ, Sutton AP (1998) Electron-energy-loss spectra and the structural stability of nickel oxide: an LSDA+U study. Phys Rev B 57(3):1505-1509. https://doi.org/10.1103/PhysRevB.57.1505

37. Sun J, Ruzsinszky A, Perdew JP (2015) Strongly constrained and appropriately normed semilocal density functional. Phys Rev Lett 115(3):036402. https://doi.org/10.1103/PhysRevLett.115.036402

38. Calderon CE, Plata JJ, Toher C, Oses C, Levy O, Fornari M, Natan A, Mehl MJ, Hart G, Buongiorno Nardelli M, Curtarolo S (2015) The AFLOW standard for high-throughput materials science calculations. Comput Mater Sci 108:233-238. https://doi. org/10.1016/j.commatsci.2015.07.019

39. Sun J, Remsing RC, Zhang Y, Sun Z, Ruzsinszky A, Peng H, Yang Z, Paul A, Waghmare U, Wu X, Klein ML, Perdew JP (2016) Accurate first-principles structures and energies of diversely bonded systems from an efficient density functional. Nature Chem 8(9):831-836. https://doi.org/10.1038/nchem.2535

40. Monkhorst HJ, Pack JD (1976) Special points for brillouin-zone integrations. Phys Rev B 13(12):5188-5192. https://doi.org/10. 1103/PhysRevB.13.5188

41. Bader RFW (1990) Atoms in molecules-a quantum theory. Oxford University Press, Oxford

42. Tang W, Sanville E, Henkelman G (2009) A grid-based bader analysis algorithm without lattice bias. J Phys Condens Matter 21(8):084204. https://doi.org/10.1088/0953-8984/21/8/084204

43. Henkelman G, Uberuaga BP, Jónsson H (2000) A climbing image nudged elastic band method for finding saddle points and minimum energy paths. J Chem Phys 113(22):9901-9904. https://doi. org/10.1063/1.1329672

44. Henkelman G, Jónsson H (1999) A dimer method for finding saddle points on high dimensional potential surfaces using only first derivatives. J Chem Phys 111(15):7010-7022. https://doi.org/10. $1063 / 1.480097$

45. Krasheninnikov AV, Lehtinen PO, Foster AS, Pyykkö P, Nieminen RM (2009) Embedding transition-metal atoms in graphene: structure, bonding, and magnetism. Phys Rev Lett. https://doi.org/ 10.1103/PhysRevLett.102.126807

46. Guo X, Huang S (2018) Tuning nitrogen reduction reaction activity via controllable Fe magnetic moment: a computational study of single $\mathrm{Fe}$ atom supported on defective graphene. Electrochim Acta 284:392-399. https://doi.org/10.1016/j.electacta.2018.07.168

47. Kattel S, Atanassov P, Kiefer B (2012) Stability, electronic and magnetic properties of in-plane defects in graphene: a first-principles study. J Phys Chem C 116(14):8161-8166. https://doi.org/ $10.1021 /$ jp2121609

48. Yazyev OV (2010) Emergence of magnetism in graphene materials and nanostructures. Rep Prog Phys 73(5):056501. https://doi. org/10.1088/0034-4885/73/5/056501

49. Ma C, Liao Q, Sun H, Lei S, Zheng Y, Yin R, Zhao A, Li Q, Wang B (2018) Tuning the doping types in graphene sheets by $N$ monoelement. Nano Lett 18(1):386-394. https://doi.org/10.1021/ acs.nanolett.7b04249

50. Tison Y, Lagoute J, Repain V, Chacon C, Girard Y, Rousset S, Joucken F, Sharma D, Henrard L, Amara H, Ghedjatti A, Ducastelle F (2015) Electronic interaction between nitrogen atoms in doped graphene. ACS Nano 9(1):670-678. https://doi.org/10. $1021 / \mathrm{nn} 506074 \mathrm{u}$

51. Wu M, Cao C, Jiang JZ (2010) Light non-metallic atom (B, N, O and F)-doped graphene: a first-principles study. Nanotechnology 21(50):505202. https://doi.org/10.1088/0957-4484/21/50/505202

52. Zhang Y-H, Chen Y-B, Zhou K-G, Liu C-H, Zeng J, Zhang H-L, Peng Y (2009) Improving gas sensing properties of graphene by introducing dopants and defects: a first-principles study. Nanotechnology 20(18):185504. https://doi.org/10.1088/0957-4484/ 20/18/185504

53. Ghosh D, Pati SK (2017) Trapping of gaseous pollutants on defective N-doped graphene. Phys Chem Chem Phys 19(1):636-643. https://doi.org/10.1039/C6CP06247D

54. Kropp T, Mavrikakis M (2019) Transition metal atoms embedded in graphene: how nitrogen doping increases $\mathrm{CO}$ oxidation activity. ACS Catal 9(8):6864-6868. https://doi.org/10.1021/acscatal. 9b01944

55. Nørskov JK, Rossmeisl J, Logadottir A, Lindqvist L, Kitchin JR, Bligaard T, Jónsson H (2004) Origin of the overpotential for oxygen reduction at a fuel-cell cathode. J Phys Chem B 108(46):17886-17892. https://doi.org/10.1021/jp047349j

56. Kattel S, Atanassov P, Kiefer B (2012) Density functional theory study of $\mathrm{Ni}-\mathrm{N}_{x} / \mathrm{C}$ electrocatalyst for oxygen reduction in alkaline and acidic media. J Phys Chem C 116(33):17378-17383. https:// doi.org/10.1021/jp3044708

57. Liu J, Lucci FR, Yang M, Lee S, Marcinkowski MD, Therrien AJ, Williams CT, Sykes ECH, Flytzani-Stephanopoulos M (2016) Tackling $\mathrm{CO}$ poisoning with single-atom alloy catalysts. J Am Chem Soc 138(20):6396-6399. https://doi.org/10.1021/jacs.6b033 39

58. Wu L, Cao X, Hu W, Ji Y, Zhu Z-Z, Li X-F (2019) Improving the oxygen reduction reaction activity of $\mathrm{FeN}_{4}$-graphene via tuning electronic characteristics. ACS Appl Energy Mater 2(9):66346641. https://doi.org/10.1021/acsaem.9b01164

59. Calle-Vallejo F, Martínez JI, Rossmeisl J (2011) Density functional studies of functionalized graphitic materials with late transition metals for oxygen reduction reactions. Phys Chem Chem Phys 13(34):15639. https://doi.org/10.1039/c1cp21228a

60. Liang W, Chen J, Liu Y, Chen S (2014) Density-functional-theory calculation analysis of active sites for four-electron reduction of $\mathrm{O}_{2}$ on $\mathrm{Fe} / \mathrm{N}$-doped graphene. ACS Catal 4(11):4170-4177. https:// doi.org/10.1021/cs501170a

61. Tang J-Y, Shen J-S, Chen L, Jiang J-W, Lu J, Zhao X, Dai G-L (2018) Investigation of carbon monoxide catalytic oxidation on vanadium-embedded graphene. Monatsh Chem 149(8):13491356. https://doi.org/10.1007/s00706-018-2181-3

62. Li Y, Zhou Z, Yu G, Chen W, Chen Z (2010) CO catalytic oxidation on iron-embedded graphene: computational quest for low-cost nanocatalysts. J Phys Chem C 114(14):6250-6254. https://doi.org/ $10.1021 /$ jp911535v

63. Xu X-Y, Xu H, Guo H, Zhao C (2020) Mechanism investigations on $\mathrm{CO}$ oxidation catalyzed by $\mathrm{Fe}$-doped graphene: a theoretical study. Appl Surf Sci 523:146496. https://doi.org/10.1016/j.apsusc. 2020.146496

64. Deng Q, Zhao L, Gao X, Zhang M, Luo Y, Zhao Y (2013) Single layer of polymeric cobalt phthalocyanine: promising low-cost and high-activity nanocatalysts for CO oxidation. Small 9(20):35063513. https://doi.org/10.1002/smll.201300652

65. Li F, Li Y, Zeng XC, Chen Z (2015) Exploration of high-performance single-atom catalysts on support $\mathrm{M} 1 / \mathrm{FeOx}$ for $\mathrm{CO}$ 
oxidation via computational study. ACS Catal 5(2):544-552. https://doi.org/10.1021/cs501790v

66. Luo M, Liang Z, Liu C, Liu M, Qi X, Chen M, Yang H, Liang $\mathrm{T}$ (2020) Theoretical calculation of different reaction mechanisms for CO oxidation on MnN3-doped graphene. ACS Omega 5(33):21203-21210. https://doi.org/10.1021/acsomega.0c02930

67. Wang M, Wang Z (2017) Single Ni atom incorporated with pyridinic nitrogen graphene as an efficient catalyst for $\mathrm{CO}$ oxidation: first-principles investigation. RSC Adv 7(77):48819-48824. https://doi.org/10.1039/C7RA06591D

68. Liu J, Jiao M, Lu L, Barkholtz HM, Li Y, Wang Y, Jiang L, Wu Z, Liu D, Zhuang L, Ma C, Zeng J, Zhang B, Su D, Song P, Xing W, Xu W, Wang Y, Jiang Z, Sun G (2017) High performance platinum single atom electrocatalyst for oxygen reduction reaction. Nat Commun 8(1):15938. https://doi.org/10.1038/ncomms15938
69. Fu Z, Yang B, Wu R (2020) Understanding the activity of single-atom catalysis from frontier orbitals. Phys Rev Lett 125(15):156001. https://doi.org/10.1103/PhysRevLett.125.156001

70. Farkas A, Zalewska-Wierzbicka K, Bachmann C, Goritzka J, Langsdorf D, Balmes O, Janek J, Over H (2013) High pressure carbon monoxide oxidation over platinum (111). J Phys Chem C 117(19):9932-9942. https://doi.org/10.1021/jp401867g

71. Tang Y, Yang Z, Dai X (2012) A theoretical simulation on the catalytic oxidation of $\mathrm{CO}$ on Pt/graphene. Phys Chem Chem Phys 14(48):16566. https://doi.org/10.1039/c2cp41441d

Publisher's Note Springer Nature remains neutral with regard to jurisdictional claims in published maps and institutional affiliations. 